\title{
Los finos del árido calizo de machaqueo en la fabricación de hormigones de altas prestaciones: datos para un tema en discusión
}

Fecha de recepción: 29-I-99

Fecha de aceptación: 23-VII-99
A.ETXEBARRIA*,J.L.RAMIREZ ,J.I.URRETA* ${ }^{*}$ J.GORBEÑA *

${ }^{(*)}$ Dres. Ingenieros Industriales. LABEIN, Centro Tecnológico. Bilbao

ESPAÑA
RESUMEN

En este articulo se trata el tema de la utilización de los áridos calizos de machaqueo, tanto gruesos como finos, en la fabricación de Hormigones de Altas Prestaciones (H.A.P). Para ello se comentan, en primer lugar, los diferentes trabajos de investigación desarrollados en este sentido a nivel internacional, para, posteriormente, centrarse en las investigaciones más recientes que, sobre este tema, se han desarrollado en España. Se presenta, asimismo, una lista de obras ejecutadas en España con H.A.P. con árido calizo y se facilita una amplia lista bibliográfica sobre el tema en discusión.

\section{SUMMARY}

This paper deals with the use of crushed limestone aggregate, fine and coarse, in High Performance Concrete (H.P.C.) manufacturing. Firstly, different international research projects about this subject are presented and following, a focus on the Spanish experience is made. In addition the paper provides a list of works made in Spain with HPC from crushed limestone aggregates and a complete bibliography section about the subject in discussion.

\section{INTRODUCCIÓN}

En la mayor parte del País Vasco, y en otras Comunidades del Estado, las canteras son de árido calizo. En la producción de árido calizo de machaqueo, principalmente en el caso de la arena, se genera una gran cantidad de finos $(<80 \mu \mathrm{m})$ difícil de eliminar. Estos finos son considerados como elemento a controlar en el hormigón convencional por lo que su porcentaje en los áridos del hormigón se encuentra actualmente limitado por las normativas internacionales: A.F.N.O.R., B.S., A.S.T.M...llegándose en la normativa española E.H.-91 a contenidos máximos del $10 \%$ al $15 \%$, dependiendo del ambiente en el que esté situada la obra.

En el caso de los Hormigones de Altas Prestaciones $\left(f_{c k} \geq 500 \mathrm{MPa}\right.$ ) la situación es similar, al menos en España, si bien, en este caso, la recomendación-limitación en cuanto al contenido de finos máximo permitido es mucho más exigente. Así, por ejemplo, en el boletín n 20 del GEHO sobre fabricación y puesta en obra del H.A.R., el contenido máximo de finos establecido para la arena es del $1 \%$, un valor imposible de obtener en la práctica real cotidiana de las canteras de árido calizo. Más aún, a un nivel prácticamente internacional se desaconseja el uso de árido fino calizo en general por dar lugar a una gran demanda de agua y por generar mezclas poco trabajables.

\section{LOS ÁRIDOS CALIZOS DE MACHAQUEO EN LOS HORMIGONES DE ALTAS PRESTACIONES: EXPERIENCIAS INTERNACIONALES}

\section{1. Árido calizo de machaqueo grueso}

Los áridos forman una parte importante de la masa de hormigón. Si se observan los volúmenes absolutos de los distintos componentes del hormigón convencional se aprecia que, aproximadamente, tres cuartas partes del mismo están constituidas por árido.

Este hecho da cierta importancia a los áridos y a sus características mecánicas por su repercusión en las propiedades del hormigón, tanto fresco como endurecido (1). 
En el caso de los hormigones convencionales, esta importancia es relativa ya que las dosificaciones de cemento y agua habitualmente empleadas confieren a la pasta de mortero unas características resistentes muy inferiores a las propias de un árido sano y compacto (2). Es por ello que la rotura en este tipo de hormigones se produce generalmente por agotamiento de la capacidad de dicha pasta, observándose así grietas en la misma, que se propagan por la masa de hormigón hasta llegar al árido grueso, al que circunvalan, sin afectarle, antes de proseguir su trayectoria (3).

En los hormigones de altas prestaciones (H.A.P.), sin embargo, las altas dosificaciones de cemento empleadas $\mathrm{y}$ las bajas relaciones $\mathrm{a} / \mathrm{c}$ admitidas generan un fuerte incremento de la capacidad resistente de la pasta de mortero. De esta forma, es ahora el árido el que primero se agota y rompe, por lo que sus características se convierten en un factor determinante del HAP, y que, por lo tanto, hay que estudiar en detalle (4) (5).

Esta circunstancia es aplicable fundamentalmente alárido grueso cuyas características son decisivas enla resistencia a compresión y el módulo de elasticidad del HAP. Las propiedades mecánicas del árido fino parecen tener mucha menos importancia (4).

Por otra parte, en la fabricación del hormigón, convencional o de altas prestaciones, el transporte del árido desde zonas alejadas hace que el producto final no resulte económicamente factible y de ahí que se imponga el ánalisis de los áridos locales.

Por todo lo expuesto anteriormente, han proliferado en los últimos años los estudios relacionados con la obtención de HAP utilizando diversos tipos de áridos gruesos y comparando resultados (3) (6-17), etc. En estos trabajos ha sorprendido siempre el buen comportamiento mecánico y de durabilidad de los áridos calizos de machaqueo, a pesar de ser los áridos que, generalmente, eran más porosos y presentaban un mayor coeficiente de absorción.

Dicho buen comportamiento lo justifican los distintos autores con diversas explicaciones, entre las que destacan las siguientes:

$\left.1^{\circ}\right)$ La textura del árido: la superficie rugosa y angular de estos áridos facilita una mejor ligazón con la pasta de cemento, fortaleciendo la zona de transición entre el árido y la pasta (7).

$\left.2^{\circ}\right)$ El coeficiente de absorción: la mayor absorción superficial de estos áridos hace que la zona de transición presente menor espesor y/o menor cantidad de agua, fenómenos ambos favorecedores para la obtención de mejores resistencias en el hormigón (18). $\left.3^{\circ}\right)$ La composición química del árido: la caliza del árido, al ser de la misma naturaleza que la cal del cemento, favorece la adherencia epitáxica árido-pasta y fortalece, así, la zona de transición entre ambos (19) (20).

Para zonas, como el País Vasco, donde la práctica totalidad de las canteras son de árido calizo, ésta debería ser una buena noticia con vistas a la fabricación de HAP, pero no es así. Mientras el árido calizo grueso $(>5 \mathrm{~mm})$ ha sido estudiado y ensayado repetidamente en la fabricación de HAP proporcionando muy buenos resultados, el árido calizo de machaqueo fino $(<5 \mathrm{~mm})$ se encuentra "proscrito" de dicha fabricación.

\section{2. Árido calizo de machaqueo fino}

En la práctica real no existe una normativa específica y de obligado cumplimiento que prohiba taxativamente la utilización del árido fino de machaqueo, pero son numerosas las normas y recomendaciones que aconsejan evitar el uso de este tipo de arenas por su excesivo contenido de finos. Este contenido se considera superfluo e innecesario en los hormigones de altas prestaciones, que ya de por sí poseen una elevada cantidad de finos por su alta dosificación de cemento y por la adición de partículas ultrafinas puzolánicas (microsílice, cenizas volantes...) y además, se añade, provoca un aumento en la demanda de agua de la mezcla, con lo cual es más difícil lograr las bajas relaciones a/c características en este tipo de hormigones (4) (19).

Otros autores priorizan el uso de las arenas rodadas sobre las de machaqueo no tanto por su menor contenido en finos (que consideran algo sin interés debido al elevado contenido de finos habitual en los HAP), como por su geometría. Dentro de esta línea destaca F. de Larrard, coautor del "modelo lineal de la compacidad máxima de mezclas de áridos" (21) que recomienda el uso de las arenas rodadas debido a la mayor compacidad global (y, por lo tanto, mayor resistencia final) que la forma esférica de sus partículas proporciona a la masa de hormigón.

En conclusión, en la fabricación de HAP la arena en general recomendada, nacional e internacionalmente, es la arena de río, rodada y con el mínimo contenido de finos posible (4) (19) (22).

A pesar de esta recomendación, no hay que olvidar, sin embargo, la existencia de diversos trabajos de investigación en los que, comparadas las arenas rodadas con las de machaqueo, éstas últimas proporcionaron iguales o mejores resultados de resistencia a compresión en el HAP.

Tenemos así el trabajo de C. Bedard y otros (9) en el que las arenas dolomíticas machacadas ensayadas dieron los mejores resultados debido a su mayor adherencia con la 
pasta de cemento y a la mayor homogeneidad que presentaba el hormigón fabricado con ellas, al tratarse de masas con árido grueso y fino procedentes de la misma roca.

También es de destacar el trabajo desarrollado por Donza y Cabrera (23) ensayando HAP fabricados con arena natural y comparando las resistencias obtenidas con las resultantes de fabricar este mismo HAP sustituyendo el $25,50,75$ y $100 \%$ de dicha arena natural por arena de granito machacada, no observándose diferencias apreciables.

En cualquier caso conviene matizar que, en el primero de los trabajos, se trataba de arenas de machaqueo relativamente gruesas, $y$, en el segundo, de arena de machaqueo lavada, que presentaba la misma curva granulométrica que la arena natural a la que sustituía, con tan sólo un $1 \%$ de finos y un módulo de finura de 3,33.

Precisamente, otra recomendación bastante habitual para las arenas a ser utilizadas en la fabricación de los HAP es que presenten un módulo de finura próximo a 3, si bien existe un estudio (12) en el que se ha concluido la no influencia de este factor en la resistencia a compresión del HAP.

En parte debido a las recomendaciones anteriormente citadas $\mathrm{y}$, en parte, debido a lo limitado de las zonas que cuentan, casi únicamente, con canteras de árido calizo (caso del País Vasco), el resultado es que, en la mayor parte de la bibliografía consultada sobre HAP, el árido fino empleado es arena natural rodada.

Como caso excepcional se presentan los HAP fabricados para soportar el fuego o las altas temperaturas. Para este tipo de hormigones es habitual emplear áridos calizos, por su mayor resistencia térmica (24) (25).

En otras ocasiones, por ej. en el caso de que la arena rodada a utilizar tenga un módulo de finura excesivamente bajo (19) (26) (27), o en el caso de que se quiera mejorar la resistencia a la abrasión del hormigón resultante (28), se pueden encontrar HAP en los que la arena es una mezcla de árido fino rodado y árido fino machacado, pero son mucho más abundantes las dosificaciones con un único tipo de arena que, en la práctica totalidad de los casos, es rodada.

Cabe anotar que, aparte de los trabajos anteriormente citados de C. Bedard (9) y Donza y Cabrera (23), no son muchas las investigaciones desarrolladas con árido fino machacado y, en general, en los trabajos realizados con este tipo de arena el objetivo planteado es la comparación entre HAP con arenas rodadas y con arenas machacadas y no el análisis en profundidad de la influencia de éstas últimas en las características del HAP fabricado.
Además, las arenas machacadas estudiadas son generalmente diabasas, dolomitas y granitos (9) (23), (29), y, rara vez, calizas, árido característico del País Vasco y de otras zonas de España.

De mayor interés para nuestros propósitos resulta el trabajo de investigación desarrollado por L. García Ballester, C. Ayats y R. Capuz (30), elaborando en Valencia un HAP de $50 \mathrm{MPa}$ utilizando únicamente áridos calizos, finos y gruesos, de distintas épocas geológicas y con distintas proporciones árido grueso/ árido fino $(\mathrm{AG} / \mathrm{AF})$.

Del trabajo realizado, los autores concluyen que la resistencia a compresión en los HAP está influenciada por la relación $\mathrm{AG} / \mathrm{AF}$, más que por las características geológicas del árido, siempre que éste no presente fisuración. Las relaciones $\mathrm{AG} / \mathrm{AF}$ que mejores resultados proporcionan son las comprendidas en el intervalo 1,32,3 , valores similares a los aplicados y recomendados por otros muchos investigadores (6) (23) (31) (32), aunque también hay autores (33) que mantienen que dicha relación apenas tiene influencia en las resistencias finales, siendo el factor clave el aseguramiento de la continuidad de la curva granulométrica, para evitar segregación.

En cuanto a las arenas, los autores señalan como, aunque éstas presentaban un contenido de partículas inferiores a los $0,16 \mathrm{~mm}$, superior al $10 \%$ máximo permitido por la norma ASTM C33-90, los hormigones obtenidos con ellas superaban los $50 \mathrm{MPa}$ de resistencia a los 28 días, definidos de partida en el diseño. Por este motivo decidieron extender el uso granulométrico de las arenas, facilitado por la ASTM C33-90, de un 10 hasta un 15\%, para las partículas menores de $160 \mu \mathrm{m}$.

Finalmente, los autores concluyen que el contenido de finos correspondiente a la fracción granulométrica $0 / 0,16$ influye sobre la resistencia, si bien es necesaria más investigación sobre la influencia que pueda tener el hecho de que el porcentaje corresponda a la subfracción $0,08 / 0,16$ o a la subfracción $0 / 0,08$.

A este respecto cabe anotar que, en la amplia bibliografia consultada, no se ha encontrado ninguna ponencia en la que se trate este último tema ni se investigue el papel de los finos de la arena de machaqueo en las resistencias de HAP.

Al igual que algunas zonas de España, otro país con gran tradición en áridos calizos es Francia y son así numerosos los trabajos desarrollados en este país con HAP elaborado únicamente con árido calizo, fino y grueso (34-39).

Pero, a decirverdad, la mayor parte de estas investigaciones no se preocupan de estudiar el árido empleado, sino las características del HAP, fresco o endurecido, fabricado 
con él, por lo que no son de gran ayuda para nuestro estudio, si bien dan una idea de las dosificaciones y proporciones empleadas con este tipo de áridos, asi como de la posibilidad de desarrollar HAP con ellos, a pesar de su mayor contenido en finos.

En este sentido, y a pesar de las recomendaciones que aconsejan el uso de arenas rodadas por su menor contenido en finos, caben destacar los trabajos descritos en (40-42), en los que se recomienda, o pone en práctica, la adición de filler calizo a la masa de HAP como una adición más (como la microsílice, por ejemplo) a fin de mejorar su trabajabilidad y compacidad; o la investigación desarrollada en [24], en la que se añade filler calizo a fin de disminuir los efectos de la reacción exotérmica de hidratación. Ahora bien, hay que anotar que se trata, en todos los casos, de un filler convenientemente controlado, de regularidad garantizada.

En cuanto al aspecto de que las mezclas de HAP con arena fina machacada exigen un mayor contenido de agua, convendría recordar el trabajo desarrollado por M. Buil y A. M. Paillière (43) en el que se observaba que, si bien los ultrafinos calizos aumentan la demanda de agua (o de superplastificante) de la masa, este aumento es bastante inferior al provocado por la adición de microsílice.

A este respecto, conviene también destacar el trabajo desarrollado por Kankkunnen y Ojanen (1) en el que se compara el efecto de diversos tipos de áridos, rodados y machacados, en la trabajabilidad del hormigón fabricado. De su investigación, los autores concluyen que el factor que más influye en la trabajabilidad es el árido grueso, cuyo incremento supone un aumento apreciable en la demanda de agua de la masa y cuya forma geométrica tiene una gran influencia en la misma.

Sin embargo, añaden, el incremento de la fracción más fina, de $0 / 0,15 \mathrm{~mm}$, tan sólo supone un ligero aumento en la demanda de agua y, de hecho, se considera necesario que esta fracción no sea excesivamente pequeña, ya que generaría una disminución de la trabajabilidad.

En este sentido, cabe destacar la comparación que los autores realizan entre el filler calizo convencional y otros dos tipos de finos (microsílice y cenizas volantes), concluyendo que el filler (10\%) y las cenizas volantes (9\%) mejoran la trabajabilidad del hormigón patrón, mientras que la microsílice (4\%), la empeora.

\section{3.- Aplicaciones prácticas con árido calizo de machaqueo, fino y grueso}

Lo que más sorprende del estudio bibliográfico realizado es que, aconsejando las recomendaciones y normas nacionales e internacionales el uso de arena rodada, y no existiendo apenas, como acaba de quedar constatado anteriormente, investigaciones que soporten el uso del árido fino calizo de machaqueo en la fabricación del HAP, en la práctica, sin embargo, se pueden encontrar numerosas realizaciones de HAP con este tipo de áridos tanto en Francia como en España.

En nuestro país, curiosamente, buena parte de las contadas realizaciones con HAP han sido ejecutadas precisamente con árido calizo, fino y grueso. Entre ellas, se destacan las siguientes, extraídas de (19) (44) (45) y (46) (Tabla 1).

TABLA 1

\begin{tabular}{|l|c|c|c|c||}
\hline \multicolumn{1}{|c|}{ OBRA } & AÑO & \%FINOS & $\begin{array}{c}\text { RESISTENCIA } \\
\text { CARACTERISTICA (MPa) }\end{array}$ & UBICACIÓN \\
\hline \hline Viaducto del Besós & 1992 & 10 & 40 & Barcelona \\
\hline Pasarelas del puerto de Málaga & 1992 & $?$ & 60 & Málaga \\
\hline Puente sobre el río Miño & 1995 & 10 & 65 & Lugo \\
\hline Puente sobre el río Guadalete (*) & 1995 & $?$ & 80 & Cádiz \\
\hline Conjunto residencial Natura Playa & 1996 & 10 & 70 & Alicante \\
\hline Dovelas túnel Guadiaro-Majaceite & 1996 & $?$ & 80 & --- \\
\hline
\end{tabular}

(") Previamente a la ejecución del puente se llevaron a cabo distintos ensayos en laboratorio con diferentes materiales: cemento, áridos, superplastificante y microsílice (47). Los resultados de resistencia y trabajabilidad obtenidos en las distintas masas presentaron pequeñas diferencias. Concretamente, en el caso de los áridos, se obtuvieron ligeras mejoras de resistencia empleando unos áridos diferentes a los calizos de machaqueo, habitualmente utilizados en la planta de fabricación. Sin embargo, considerando lo poco significativo de estas mejoras, asi como la necesidad de llevar a cabo ciertos ajustes para el almacenamiento de los nuevos áridos, se decidió utilizar los calizos de machaqueo, aptos para la fabricación del HAP especificado para el puente, y, por otra parte, ya muy conocidos por el fabricante de hormigón, debido a su uso continuado y a los periódicos controles de calidad realizados en el mismo. 
Como vemos, en tres de las cinco realizaciones señaladas no se tienen datos acerca del contenido de finos en las arenas empleadas pero, dado que se trata de obras ejecutadas sin especiales requisitos para los áridos, es lógico suponer que se trata de arenas con contenidos de finos en torno al $10 \%$, como en las otras tres obras, en las que sí se dispone de este dato, o quizás incluso superiores.

Pero el hecho que nos parece interesante destacar es que, tal y como demuestran estas realizaciones prácticas, es posible obtener en obra un HAP de hasta $80 \mathrm{MPa}$ utilizando únicamenteárido calizo de machaqueo y eso aun tratándose de arenas con contenidos relativamente altos $(10 \%)$ de finos.

A la vista de estas realizaciones no cabe sino meditar sobre el comentario que el boletín no 20 del GEHO (19) hace en su artículo 1.1.3 Áridos, pág. 9:

“...El empleo de arenas de machaqueo no es recomendable por dar lugar a una gran cantidad de agua y por generar mezclas poco trabajables; no obstante, en caso de carencia de arena de río, pueden emplearse aquellas después de estudiar previamente su comportamiento mediante ensayos".

Comentario que es, por otra parte, muy similar al que la norma canadiense CSA A23.1-94 hace en su capítulo 29, sección 29.2.

\section{LOS ÁRIDOS CALIZOS DE MACHAQUEO EN LOS HORMIGONES DE ALTAS PRESTACIONES: INVESTIGACIONES RECIENTESENESPAÑA}

En España caben señalar los trabajos de investigación desarrollados en Galicia y en el País Vasco en los últimos años.

En Galicia, la ETS de Ingenieros de Caminos, Canales y Puertos de La Coruña ha desarrollado un completo estudio para fabricar HAP con el empleo de diversas combinaciones de áridos de machaqueo locales, fundamentalmente granito, caliza y cuarcita, tanto para el árido grueso como para el fino (48).

Como resultado de este estudio se ha demostrado la viabilidad de fabricar HAP(resistencias medias máximas a 28 días $=90 \mathrm{MPa}$ ) con árido machacado gallego, habiendo desarrollado los investigadores métodos de dosificación y secuencias de fabricación ad-hoc destinados a mejorar el comportamiento global de estos hormigones.

En el País Vasco, en el Centro Tecnológico LABEIN, hemos llevado a cabo un trabajo de investigación encaminado a desarrollar HAP utilizando únicamente árido calizo local, fino y grueso, con contenidos de finos (partículas $<80 \mu \mathrm{m}$ ) de hasta un 20\% (49-51).

El trabajo se ha desarrollado en 4 fases correlativas que nos llevaron, por aproximaciones sucesivas, al resultado final. Estas 4 fases fueron:

\section{Fase previa}

Selección de componentes y determinación del campo experimental.

\section{Fase A}

Determinación del tipo y dosificación de cemento.

\section{Fase B}

Determinación de la dosificación de microsílice y superplastificante, en peso de cemento.

\section{Fase C}

Determinación de la influencia del contenido en finos en las resistencias de los hormigones elaborados.

En total se llevaron a cabo unas 100 amasadas de hormigón, de cada una de las cuales se elaboraron 12 probetas cilíndricas, de $10 \times 20 \mathrm{~cm}$, y que fueron ensayadas a compresión a 3, 7, 28 y 91 días, respectivamente. El análisis de los resultados de resistencia obtenidos fue llevado a cabo mediante la utilización de técnicas estadísticas basadas en el Diseño de Experimentos.

Concretamente se utilizó la metodología del Diseño de Mezclas que nos permitió obtener una ecuación del modelo experimental en la que se relaciona la resistencia a compresión con los distintos componentes de la mezcla y que, por lo tanto, nos permite definir las dosificaciones óptimas desde un punto de vista de máxima resistencia a compresión.

Además, y como conclusión del estudio, se ha confirmado la posibilidad de fabricar HAP con resistencias medias a 91 días superiores a los $100 \mathrm{MPa}$ utilizando únicamente árido calizo machacado, fino y grueso, con independencia del contenido en finos (entre $8 \%$ y $20 \%$ ) que dicho árido presente.

\section{CONCLUSIONES}

A la vista de las distintas investigaciones realizadas parece claro que el árido de machaqueo, tanto grueso como fino, es perfectamente válido para la elaboración de HAP.

Esta afirmación queda corroborada y avalada por las numerosas obras ejecutadas con HAP elaborado con este tipo de áridos, fundamentalmente en Francia y España. 


\section{BIBLIOGRAFÍA}

(1)H. Kankkunen, P.Ojanen. “Concrete rheology and compaction". 1992. Nordic concrete research. Publication No 11 . The Nordic concrete federation. 1/1992. Pág.100-109.

(2) S.L. Sarkar. "The importance of microstructure in evaluating concrete". 1994. Canmet, Advances in concrete technology. Pág. 125-160.

(3) G. González Isabel. "Hormigón de alta resistencia". 1993. Instituto técnico de materiales y construcciones, Intemac.

(4) FIP/CEB. "High strength concrete. State of the art report". 1990. Bulletin d'information 197.

(5) O.E. Gjoerv. "High strength concrete". 1994. Canmet, Advances in concrete technology. Pág.18-92.

(6) M. Fdez. Cánovas. "Hormigones de alta resistencia". 1988. Cemento-Hormigón nº 658.

(7) R.C. Kriesel, C. W. French. "Durability of HPC". 1995. Structural faults and repair congress, 1995.

(8) M.R. Taylor, F.D. Lydon, B.I.G. Barr. “Mix proportions for HSC”. 1996. Construction and building materials, Vol. 10, Nº, pp 445-450.

(9) C. Bedard, G. Ballivy, P.C. Aïtcin. "Role des caracteristiques physico-mecaniques des granulats sur la résistance en compression de bétons à trés haute résistance”. 1984. Bulletin de l'association internationale de géologie de l'ingenieur Nº 30. Págs. 187-191.

(10) P.C. Aïtcin, P.K. Mehta. "Effect of coarse aggregate characteristics on mechanical properties of HSC". 1990. ACI Materials Journal, Vol.87., N², Págs. 103-107.

(11) P.C. Aïtcin. S.L.Sarkar, Y. Diatta. "Microstructural study of different types of very HSC". 1987. Materials Research Society Symposiumproceeding. Vol.85, Págs. 261-272.

(12) A.A. Ramezanianpour. "Selection of materials and mix proportions for HSC". 1993. Proceedings of the 3rd International Symposium on HSC, Norway. Vol. 2, Págs. 904-912.

(13) J.E. Jaí, J. Calavera, J. Fdez. Gómez. "Proyecto de dosificación de un HAR”. 1992. X Congreso internacional del hormigón preparado. $\mathrm{VI} / \mathrm{A}-1$.

(14) I. Schrage, R. Springeschmid. "Creep and shrinkage data of HSC". 1996. Proceedings of the 4th International symposium on utilization of HSC/HPC. Paris, Francia. Vol.2. Págs. 331-338.

(15) N. Han, J.C. Walraven. "Creep and shrinkage of young HSC". 1996. Proceedings of the 4th International symposium on utilization of HSC/HPC. Paris, Francia. Vol.2. Págs. 339-348.

(16) J.F. Cubaynes, G. Pons. "Influence of the type of coarse aggregates on shrinkage and creep of HSC". 1996. Proceedings of the 4th International symposium on utilization of HSC/HPC. Paris, Francia. Vol.2. Pag. 397-404.

(17) R.C. Chen, R.L. Carrasquillo, D.W. Fowler. "Behaviour of HSC under uniaxial and biaxial compression". 1987. HSC Seminar course manual. SCM-15 (87). ACI-87-14. Págs. 251-277.

(18) P.K. Mehta, P.C. Aïtcin. "Microstructural basis of selection of materials and mix proportions for HSC". 1990. Proceedings of the 2nd International Symposium on utilization of HSC. California, USA. 1990.

(19) Grupo español del hormigón. Comisión I, G.T.I/2. "Hormigón de alta resistencia, fabricación y puesta en obra". 1997. Boletín n²0, GEHO.

(20) M.P. Alaejos, M. Fdez. Cánovas. "Hormigón de alta resistencia: dosificación y propiedades mecánicas”. 1995. Centro de estudios y experimentación de obras públicas, CEDEX, M49.

(21)F. De Larrard. "Formulation et propietés des bétons à trés hautes performances". 1988. Rapport de recherche LPC No 149. Laboratoirc central des ponts et chaussées.

(22) ACI 363R-84 "State of the art report on HSC". 1987. Seminar course manual on HSC. SCM-15 (87). ACI. Pag. 1-47.

(23) H. A. Donza, O. A. Cabrera. "The influence of kinds of fine aggregate on mechanical properties of HSC". 1996. Proceedings of the 4th International Symposium on utilization of HSC/HPC. Paris, Francia. Volumen 2. Págs. 153-160.

(24) A.N. Noumowe, P. Clasters, G. Debicki, J.L. Costaz. "Thermal stresses and water vapour pressure of HPC at high temperature". 1996. Proceedings of the 4th International symposium on utilization of HSC/HPC. Paris, Francia. 1996. Volumen 2. Págs. 561-570.

(25) R. Felicetti, P.G. Gambarova, G.P. Rosati, F. Corsi, G. Giannuzzi “"Residual mechanical properties of HSC subjected to high temperatures cycles". 1996. Proceedings of the 4th International symposium on utilization of HSC/HPC. Paris, Francia. 1996. Volumen 2. Págs. 579-588. (26) A.E. Fiorato. "Hormigón preparado de alta resistencia: propiedades para el diseño y la construcción". 1992. X Congreso internacional del hormigón preparado. VI/A-5.

(27)K. Goto, K. Kuroha, S. Namiki, H. Jinnai. "Experimental study on high strength cast-in-place concrete with 100 MPa". 1996. Proceedings of the 4th International symposium on utilization of HSC/HPC. Paris, Francia. Vol.2. Págs. 125-134.

(28) O.E.Gjoerv, T. Baerland, H.R. Roenning. "HSC for highway pavements and bridge decks". 1987. Proceedings of the $1 \mathrm{st}$ International symposium on utilization of HSC. Noruega, 1987. Tapir publishers. Págs. 111-122.

(29) M.Y.L. Chew. "Utilization of HSC in Singapore". 1993. Proceedings of the 3rd International symposium on utilization of HSC. Noruega, 1993. Volumen 2. Págs. 678-690.

(30) L. García, C. Ayasts, R. Capuz. "Los áridos calizos en los hormigones de alta resistencia”. 1996. Hormigón N² 27. Áridos.

(31) C. Ellis. "The rheological and mechanical properties of water reduced superplasticised HSC". 1996. Proceedings of the 4th International Symposium on utilization of HSC/HPC. Paris, Francia.Volumen 2. Págs. 273-280.

(32) P. Alaejos. "Hormigones de alta resistencia: dosificación, puesta en obra y propiedades". 1995. Civil Engineering European courses. Madrid, España.

(33) J.M. Gálligo, M.P. Alaejos. "Hormigón de alta resistencia. Estado actual de conocimientos". 1990. Centro de estudios y experimentación de obras públicas, CEDEX, M19.

(34) F. de Larrard, A. Belloc. "Are small aggregates really better than coarser ones for making high strength concrete?". 1991. LCPC. Technical note.

(35) V. Waller, F. de Larrard, P. Roussel. "Modelling the temperature rise in massive HPC structures". 1996. Proceedings of the 4th International Symposium on utilization of HSC/HPC. París, Francia. Volumen 2. Págs. 415-421. 
(36) V. Baroghel-Bouny, J. Godin, J. Gawsewitch. "Microstructure and moisture properties of HPC". 1996. Proceedings of the 4th International Symposium on utilization of HSC/HPC. Paris, Francia. Volumen 2. Págs. 451-461.

(37) F. de Larrard, G. Gillet, B. Canitrot. "Preliminary HPC mix-design study for the 'Grand Viaduc de Millau': an example of LCPC's approach". 1996. Proceedings of the 4th International symposium on utilization of HSC/HPC. Paris, Francia. 1996. Volumen 3. Págs. 13231331.

(38) C. Levy, J.P. Le Boulicaut. "HPC supplied by a network of ready-mix concrete plants". 1993. Yves Malier. HPC, from material to structure. E \& FN SPON, 1993. Págs. 63-84.

(39) M. Lorrain. "Bond properties of HPC”. 1993. Yves Malier. HPC, from material to structure. E \& FN SPON, 1993. Págs. 127-144.

(40) Rilem TC 158-AH. "The role of admixtures in high performance concrete". 1997. Materials and structures. March 1997. Págs. 47-53.

(41)F. de Larrard. "HPC: from laboratory to practical utilization". 1995. RILEM. Concrete technology: New trends, Industrial applications.

(42) L. Granger, P. Acker. "What kind of drying creep for HSC/HPC?". 1996. Proceedings of the 4th International symposium on utilization of HSC/HPC. Paris, Francia. Vol.2. Págs. 357-366.

(43) M. Buil, A.M. Paillière. "Utilization des fillers ultrafines dans les bétons". 1982. LCPC.

(44) ANEHFOP. “Hormigón de alta resistencia en Galicia”. 1995. Hormigón Vol. IX, № 24. Diciembre 1995. Págs. 22-26.

(45) R. Bueno. "El hormigón del Viaducto del Besós en Barcelona". 1992. Cemento y Hormigón n 709. Págs. 1041-1056.

(46) F. Regalado. "Natura Playa de Alicante. Primer edificio que se construye en España con un HAR H-700". 1997. Revista CementoHormigón No774. Agosto 1997.

(47) J.M. Delgado, C.S.Oteo, F. Hué. "The Guadalete river: the first Spanish experience on high strength pre-cast concrete". 1996. Proceedings of the 4th International symposium on utilization of HSC/HPC. Paris, Francia. 1996. Volumen 3. Págs. 1417-1426.

(48) F.J. Macías, F. Martínez, C. Vázquez. “Obtención de un hormigón de alta resistencia mediante el empleo de materiales gallegos: selección del árido, dosificaciones y propiedades mecánicas”. 1998. Revista Hormigón y Acero, n² 207. Págs. 11-20.

(49) A. Etxebarria. "Influencia de la variabilidad del contenido en finos de los áridos calizos de machaqueo en el desarrollo de hormigones de alta resistencia". 1998. Tesis Doctoral. Centro Tecnológico LABEIN.

(50) J. Gorbeña, A. Etxebarria, J.I. Urreta, J.L. Ramirez. “Aplicación del diseño de experimentos en la dosificación de un hormigón de altas prestaciones" $1998.1^{\text {er }}$ Symposium nacional de hormigón de altas prestaciones. Págs. 121-130.

(51) J.I. Urreta, A. Etxebarria, J.L. Rámirez, J. Gorbeña. "Influencia de la variabilidad del contenido en finos de los áridos calizos de machaqueo en las resistencias de los hormigones de altas prestaciones". 1998. $1^{\text {er }}$ Symposium nacional de hormigón de altas prestaciones

\section{Nota de la Redacción}

La Instrucción de Hormigón Estructural (EHE) aprobada por REAL DECRETO 2661/1998 de 11 de diciembre, en su articulado, al referirse a los áridos a emplear en el hormigón, permite el empleo de árido fino procedente de machaqueo de rocas calizas que contengan hasta un máximo del $15 \%$ de partículas que pasen por el tamiz de $0,063 \mathrm{~mm}$ para obras sometidas a las clases generales de exposición I, IIa y IIb y no sometidas a ninguna clase específica de exposición.

La misma Instrucción en su Anejo 11 "Hormigones de alta resistencia", al referirse a los áridos a emplear en estos hormigones, de resistencia característica fck comprendida entre 50 y $100 \mathrm{MPa}$, dice textualmente:

"Para la fabricación de hormigones de alta resistencia se emplearán áridos con propiedades mecánicas idóneas, ya que seran rodados o procedentes de rocas machacadas de alta calidad.

Desde el punto de vista mineralógico los áridos recomendables son los basaltos, cuarcitas, riolitas, sienitas ofitas y calizas de buena calidad, con densidades superiores a $2,60 \mathrm{~kg} / \mathrm{dm}^{3}$. Se recomienda que el coeficiente de desgaste de Los Ángeles no sea superior a 25.

Los áridos deben presentar un grado de limpieza muy elevado con el fin de asegurar una buena adherencia con la matriz cementante.

Es recomendable que el árido fino o arena tenga un módulo de finura próximo a 3. Se recomienda que el árido grueso tenga un tamaño no superior a $20 \mathrm{~mm}$ y que su coeficiente de forma no sea inferior a 0,25 " 